CERN-PH-TH-2004-207, DESY 04-200, Edinburgh 2004/28, FERMILAB-PUB-04-298-T, PSI-PR-04-12

\title{
Pair production of scalar leptoquarks at the LHC
}

\author{
M. Krämer \\ School of Physics, The University of Edinburgh, Edinburgh EH9 3JZ, UK* \\ T. Plehn \\ Theory Division, CERN, \\ 1211 Geneva 23, Switzerland \\ and \\ Max Planck Institut für Physik, \\ 80805 München, Germany \\ M. Spira ${ }^{\dagger}$ \\ Paul Scherrer Institut PSI, CH-5232 Villigen PSI, Switzerland \\ P.M. Zerwas \\ Deutsches Elektronen-Synchrotron DESY ${ }^{\ddagger}$, \\ D-22603 Hamburg, Germany \\ and \\ Fermi National Accelerator Laboratory, \\ P.O.Box 500, Batavia IL 60510, USA
}

(Dated: February 2, 2008)

\begin{abstract}
Theoretical predictions for the production cross sections of leptoquarks at the CERN LHC are presented including higher-order QCD corrections. These corrections reduce the dependence on the renormalization/factorization scales significantly. Moreover, they are required to exploit the leptoquark mass reach of the LHC experiments. In this sequel to an earlier analysis performed for the Tevatron collider we extend the leptoquark analysis to the LHC energy.
\end{abstract}

PACS numbers: 12.38.Bx, 12.60.-i, 14.80.-j

\footnotetext{
* present address: Institut für Theoretische Physik E, RWTH Aachen, D-52056 Aachen, Germany.

$\dagger$ Supported in part by the Swiss Bundesamt für Bildung und Wissenschaft.

$\ddagger$ Permanent address.
} 
Leptoquarks [1] have been searched for in the past at all high energy colliders operating at the energy frontier. If the Yukawa couplings for scalar leptoquark couplings to quarks and leptons of the first and second generation [on which we will focus in this report] is kept at small values, as required by low-energy precision experiments [2], the most stringent model-independent bounds on leptoquark masses, for branching ratios $B R=1$ to charged leptons, have been set with $M_{L Q} \geq 230 \mathrm{GeV}$ and $241 \mathrm{GeV}$ by the FNAL Tevatron CDF experiment for first and second generation leptoquarks, respectively, and correspondingly with 238 and $186 \mathrm{GeV}$ by the D0 experiment as reported recently in Ref. [3]; these recent individual values come close to the earlier combined limit of $242 \mathrm{GeV}$ for the first generation in Ref. [4]. The D0 and CDF experiments have also searched for leptoquarks decaying into quark and neutrino final states, resulting in a lower mass limit of $95 \mathrm{GeV}$ and $117 \mathrm{GeV}$, respectively, in these decay channels [5]. Bounds of $290 \mathrm{GeV}$ for the first generation could be set by the Zeus [6] and the H1 experiments [7] in direct electron-quark formation at DESY's HERA collider for Yukawa couplings of size 0.1 and electromagnetic strength, respectively. A limit of $300 \mathrm{GeV}$ is expected to be reached finally also by the Tevatron at the end of Run II [8].

The search for these novel particles will be continued soon at the CERN LHC. Preliminary feasibility studies by the LHC experiments ATLAS [9] and CMS [10] indicate that clear signals can be established for masses up to about $M_{L Q} \simeq 1.3$ to $1.4 \mathrm{TeV}$ for first and second generation scalar leptoquarks, with a final reach of presumably $1.5 \mathrm{TeV}$.

In this Brief Report we present the cross section for pair production of scalar leptoquarks at the LHC including next-to-leading order QCD corrections. ${ }^{1}$ The results are based on the calculation presented earlier in Ref. 11] for Tevatron energies. The higher-order corrections must be included in the theoretical predictions for the production cross sections in order to reduce the strong dependence on the renormalization and factorization scales which the lowest-order Born term calculation is bedevilled with. Moreover, for the standard choice of the common renormalization/factorization scale near the leptoquark mass, the cross section is increased by the QCD corrections and the mass range that can be probed experimentally, is extended correspondingly.

The basic processes for the production of leptoquark pairs at the LHC are gluon-gluon fusion and quark-antiquark annihilation:

$$
\begin{aligned}
& g+g \rightarrow L Q+\overline{L Q} \\
& q+\bar{q} \rightarrow L Q+\overline{L Q}
\end{aligned}
$$

as shown in Fig. 1. In the pointlike limit, the gluon-leptoquark interactions are determined by the non-abelian $\mathrm{SU}(3)_{\mathrm{C}}$ gauge symmetry of scalar QCD so that the theoretical predictions for the pair production of scalar leptoquarks are parameter-free in the first two generations for which Yukawa terms can safely be neglected [12]:

$$
\begin{aligned}
\hat{\sigma}_{g g}^{\mathrm{LO}} & =\frac{\alpha_{\mathrm{s}}^{2} \pi}{96 \hat{s}}\left[\beta\left(41-31 \beta^{2}\right)+\left(18 \beta^{2}-\beta^{4}-17\right) \log \frac{1+\beta}{1-\beta}\right] \\
\hat{\sigma}_{q \bar{q}}^{\mathrm{LO}} & =\frac{\alpha_{\mathrm{s}}^{2} \pi}{\hat{s}} \frac{2}{27} \beta^{3},
\end{aligned}
$$

\footnotetext{
${ }^{1}$ Note that QCD corrections to the production of vector leptoquarks are not under proper theoretical control as vector leptoquarks are described by a non-renormalizable effective theory.
} 
where $\beta=\left(1-4 M_{L Q}^{2} / \hat{s}\right)^{1 / 2}$ denotes the leptoquark velocity and $\hat{s}^{1 / 2}$ the invariant energy of the subprocess. As expected, for low leptoquark masses gluon fusion is by far the dominant production mechanism. Quark-antiquark annihilation becomes important for larger masses, providing $30 \%$ of the production cross section for leptoquark masses of about $1.5 \mathrm{TeV}$, as will be proved later in detail.

The calculation of the QCD radiative corrections for the total cross section of leptoquark production

$$
\sigma[p p \rightarrow L Q+\overline{L Q}]=\sigma_{g g}+\sigma_{q \bar{q}}+\sigma_{g q}
$$

including virtual effects, gluon bremsstrahlung and initial-state parton splittings at $\mathcal{O}\left(\alpha_{\mathrm{s}}\right)$ has been described in Ref. [11, 13] in detail. In the following we define the calculational scheme and the physical input parameters chosen for the present numerical analysis. The renormalization of the strong coupling $\alpha_{\mathrm{s}}(\mu)$ and the factorization of initial-state collinear singularities at $\mu$ are performed in the $\overline{\mathrm{MS}}$ scheme. The top quark and the leptoquark are decoupled from the running of $\alpha_{\mathrm{s}}(\mu)$. For the calculation of the $p p$ cross section we have adopted the CTEQ6L1 and CTEQ6M 14] parton distribution functions at LO and NLO, corresponding to $\Lambda_{5}^{\mathrm{LO}}=165 \mathrm{MeV}$ and $\Lambda_{5}^{\overline{\mathrm{MS}}}=226 \mathrm{MeV}$ at the one- and two-loop level of the strong coupling $\alpha_{\mathrm{s}}(\mu)$, respectively.

The QCD corrections strongly affect the parton cross sections near the production threshold, in particular in $g g$ fusion which rises steeply with energy. (i) Sommerfeld rescattering, i.e. Coulombic gluon exchange between the final-state partons, is singular near threshold [15] in the velocity of the produced leptoquarks, thus removing the phase-space velocity factor $\beta$ from the cross sections for S-waves and damping the phase-space suppression $\beta^{3} \rightarrow \beta^{2}$ for P-waves. These corrections are positive in the attractive color-neutral singlet channels and negative in the repulsive color-octet channels. For gluon initial states the color-singlet channels dominate, for quark-antiquark initial states the color-octet channels. The gluonexchange ladders can be resummed [16], giving rise to the threshold correction factors 17] $F_{t h}^{1,8}=1 \pm \frac{1}{2} x_{1,8} \rightarrow x_{1} /\left(1-e^{-x_{1}}\right)$ and $x_{8} /\left(e^{x_{8}}-1\right)$ with $x_{1}=\frac{4}{3} \frac{\pi \alpha_{s}}{\beta}$ and $x_{8}=\frac{1}{6} \frac{\pi \alpha_{s}}{\beta}$ for singlet and octet channels, respectively. (ii) In addition, large initial-state gluonic bremsstrahlung corrections of the type $\log ^{2} \beta$ and $\log \beta$ emerge, where the universal double logarithm can be exponentiated. The perturbative expansion of the total parton cross section can be expressed in terms of scaling functions,

$$
\hat{\sigma}_{i j}=\frac{\alpha_{\mathrm{s}}^{2}\left(M_{L Q}^{2}\right)}{M_{L Q}^{2}}\left\{f_{i j}^{B}(\beta)+4 \pi \alpha_{\mathrm{s}}\left(M_{L Q}^{2}\right)\left[f_{i j}^{V+S}(\beta)+f_{i j}^{H}(\beta)\right]\right\}_{[i, j=g, q]},
$$

where we define the Born term cross sections of the parton subprocesses by $f^{B}$ and the QCD virtual+soft and hard gluon corrections by $f^{V+S / H}$. The scaling functions depend on the invariant parton energy $\hat{s}^{1 / 2}$ through $\beta$. The expressions $f^{B}$ and $f^{V+S / H} / f^{B}$ near threshold, $\beta \ll 1$,

$$
\begin{array}{ll}
f_{g g}^{B}=\frac{7 \pi \beta}{384} & f_{q \bar{q}}^{B}=\frac{\pi \beta^{3}}{54} \\
f_{g g}^{V+S} / f_{g g}^{B}=\frac{11}{336 \beta} & f_{q \bar{q}}^{V+S} / f_{q \bar{q}}^{B}=-\frac{1}{48 \beta} \\
f_{g g}^{H} / f_{g g}^{B}=\frac{3}{2 \pi^{2}} \log ^{2}\left(8 \beta^{2}\right)-\frac{183}{28 \pi^{2}} \log \left(8 \beta^{2}\right) & f_{q \bar{q}}^{H} / f_{q \bar{q}}^{B}=\frac{2}{3 \pi^{2}} \log ^{2}\left(8 \beta^{2}\right)-\frac{107}{36 \pi^{2}} \log \left(8 \beta^{2}\right)
\end{array}
$$


can be derived from Ref. [13] by choosing the mass $M_{L Q}$ as renormalization and factorization scale. The scaling functions for $q g$ initial states are neither linearly nor logarithmically singular at threshold.

For high energies the NLO parton cross sections approach non-zero limits asymptotically in contrast to the $\sim 1 / \hat{s}$ scaling behavior of the Born cross sections. The asymptotic NLO parton cross sections read in the nomenclatura of the preceding paragraph:

$$
f_{g g}^{H}=\frac{2159}{43200 \pi}, \quad f_{q g}^{H}=\frac{2159}{194400 \pi} .
$$

The ratio between the gluon-fusion and Compton term is large, $9: 2$, which is a consequence of the large color charges of the gluons compared to the quarks.

The scale dependence of the theoretical prediction is reduced significantly when higher order QCD corrections are included. This is demonstrated in Fig. 2 where we compare, for an intermediate leptoquark mass of $1 \mathrm{TeV}$, the dependence on the common renormalization/factorization scale $\mu$ at the leading and next-to-leading order of the total cross section. For the comparison of the LO and NLO results, we have calculated all quantities [i.e. the partonic cross sections, $\alpha_{\mathrm{s}}(\mu)$ and the parton densities] consistently in leading and next-to-leading order. The scale dependence of the leading-order cross section is steep and monotonic. At next-to-leading order the scale dependence is strongly reduced, and the NLO cross section runs through a broad maximum near $\mu \approx M_{L Q} / 4$ which stabilizes the NLO prediction very effectively.

The QCD radiative corrections enhance the cross section for the production of leptoquarks in the vicinity of the standard scale choice $\mu \approx M_{L Q}$, see Fig. 3 . The $K$-factors, $K=\sigma_{\mathrm{NLO}} / \sigma_{\mathrm{LO}}$, with all quantities in the numerator and denominator calculated in NLO and LO, respectively, are displayed in Table I] [Note that $\sigma_{q g}$ is only the remnant of the $q g$ cross section after the collinear singularities are subtracted via mass factorization; overcompensation can give rise to negative values and $\sigma_{q g}$ must not be interpreted as a physical cross section.] The $K$-factors range from $K \approx 1.5$ at $M_{L Q} \approx 200 \mathrm{GeV}$ up to $K \approx 1.9$ at the upper end of the LQ mass reach of $\approx 1500 \mathrm{GeV}$ at LHC. The leptoquark results coincide with the cross sections for the production of squark-antisquark pairs in the limit of large gluino mass [13, 18, 19] after the small $(<0.5 \%)$ contributions from rescattering diagrams involving four-squark self-couplings are eliminated. This comparison provides us with an independent check of the present numerical analysis. [Agreement with the scalar cross sections for low-mass leptoquarks in the compilation of Ref. [20] is only of limited value as the $K$-factors presented in Ref. 20] have been obtained by using the NLO code of the calculation Ref. [11].] Despite the sizable corrections at the scale $\mu \approx M_{L Q}$, the moderate scale dependence of the NLO result implies a reliable perturbative expansion. ${ }^{2}$

To study the uncertainty in the prediction of the cross section inferred by the parametrization of the parton densities, we have repeated the calculation using the MRTS2002 parametrization [23]. The difference between the NLO results based on MRST2002 and

\footnotetext{
${ }^{2}$ For the production of Higgs bosons in gluon-gluon fusion, NLO $K$-factors have been found in the range between 1.5 and 2, cf. Ref. 21], but they are modified only by another $20 \%$ in NNLO 22]. From this example we may infer that also the isomorphic perturbative QCD expansion in leptoquark production is expected to converge sufficiently fast and that the NLO results are of physical significance.
} 
CTEQ6, displayed in Table 【 increases with increasing leptoquark mass due to the uncertainty in the gluon distribution at large $x$, but does not exceed $10 \%$ in the experimentally accessible mass range up to $M_{L Q} \simeq 1.5 \mathrm{TeV}$.

In conclusion. As anticipated from many calculations performed in the past, the NLO analysis stabilizes the theoretical prediction of the production cross section for leptoquarks. Moreover, for the standard choice of the renormalization and factorization scales near the leptoquark mass, the higher order corrections increase the cross section so that Born calculations, performed for the same scale of QCD coupling and parton densities, provide us with a conservative lower limit. By the same token, the NLO corrections shift the LQ mass limit upward by an amount of about $100 \mathrm{GeV}$ at the upper end of the LQ mass spectrum that can be probed at LHC.

\section{Acknowledgments}

We thank Simona Rolli for discussions on leptoquark production at the hadron colliders Tevatron and LHC that have initiated this study at the FNAL Tev4LHC Workshop. P.M. Zerwas thanks the Fermilab Theory Group for the warm hospitality during an extended visit.

[1] W. Buchmüller, R. Rückl and D. Wyler, Phys. Lett. B 191, 442 (1987) [Erratum-ibid. B 448, 320 (1999)]; see also B. Schrempp and F. Schrempp, Phys. Lett. B 153 (1985) 101.

[2] J. Kalinowski, R. Rückl, H. Spiesberger and P. M. Zerwas, Z. Phys. C 74, 595 (1997) arXiv:hep-ph/9703288.

[3] S. M. Wang [CDF Collaboration], arXiv:hep-ex/0405075.

[4] C. Grosso-Pilcher, G. Landsberg and M. Paterno [CDF Collaboration], arXiv:hep-ex/9810015.

[5] V. M. Abazov et al. [D0 Collaboration], Phys. Rev. Lett. 88, 191801 (2002) arXiv:hep-ex/0111047; D. Acosta et al. [CDF Collaboration], arXiv:hep-ex/0410076.

[6] S. Chekanov et al. [ZEUS Collaboration], Phys. Rev. D 68, 052004 (2003) arXiv:hep-ex/0304008.

[7] C. Adloff et al. [H1 Collaboration], Phys. Lett. B 523, 234 (2001) arXiv:hep-ex/0107038.

[8] S. Rolli, proceedings, Tev4LHC Workshop, FNAL 2004.

[9] V. A. Mitsou, N. C. Benekos, I. Panagoulias and T. D. Papadopoulou, proceedings, Conference on Physics at the LHC, Vienna, Austria, 13-17 July 2004, arXiv:hep-ph/0411189 (for publication in Czech. J. Phys. 54 (2004)).

[10] S. Abdullin and F. Charles, Phys. Lett. B 464, 223 (1999) arXiv:hep-ph/9905396.

[11] M. Krämer, T. Plehn, M. Spira and P. M. Zerwas, Phys. Rev. Lett. 79, 341 (1997) arXiv:hep-ph/9704322.

[12] J. A. Grifols and A. Mendez, Phys. Rev. D 26 (1982) 324; I. Antoniadis, L. Baulieu and F. Delduc, Z. Phys. C 23 (1984) 119; E. Eichten, I. Hinchliffe, K. D. Lane and C. Quigg, Rev. Mod. Phys. 56 (1984) 579 [Addendum-ibid. 58 (1986) 1065]; G. Altarelli and R. Rückl, Phys. Lett. B 144 (1984) 126; S. Dawson, E. Eichten and C. Quigg, Phys. Rev. D 31, 1581 (1985); J. Blümlein, E. Boos and A. Kryukov, Z. Phys. C 76, 137 (1997) arXiv:hep-ph/9610408. 
[13] W. Beenakker, M. Krämer, T. Plehn, M. Spira and P. M. Zerwas, Nucl. Phys. B 515, 3 (1998) arXiv:hep-ph/9710451.

[14] J. Pumplin, D. R. Stump, J. Huston, H. L. Lai, P. Nadolsky and W. K. Tung, JHEP 0207, 012 (2002) arXiv:hep-ph/0201195; D. Stump, J. Huston, J. Pumplin, W. K. Tung, H. L. Lai, S. Kuhlmann and J. F. Owens, JHEP 0310, 046 (2003) arXiv:hep-ph/0303013.

[15] J. Schwinger, Particles, Sources and Fields, Addison-Wesley, Reading, 1989; J. Jersak, E. Laermann and P.M. Zerwas, Phys. Rev. D25 (1982) 1218 [Erratum-ibid. D36 (1987) 310].

[16] A. Sommerfeld, Atombau und Spektrallinien, Vieweg, Braunschweig, 1939.

[17] V. S. Fadin, V. A. Khoze and T. Sjöstrand, Z. Phys. C 48, 613 (1990).

[18] W. Beenakker, R. Höpker, M. Spira and P. M. Zerwas, Phys. Rev. Lett. 74, 2905 (1995) arXiv:hep-ph/9412272.

[19] W. Beenakker, R. Höpker, M. Spira and P. M. Zerwas, Nucl. Phys. B 492, 51 (1997) arXiv:hep-ph/9610490.

[20] J. Blümlein, E. Boos and A. Kryukov, arXiv:hep-ph/9811271 (unpublished).

[21] D. Graudenz, M. Spira and P. M. Zerwas, Phys. Rev. Lett. 70, 1372 (1993); M. Spira, A. Djouadi, D. Graudenz and P. M. Zerwas, Nucl. Phys. B 453, 17 (1995) arXiv:hep-ph/9504378.

[22] R. V. Harlander and W. B. Kilgore, Phys. Rev. Lett. 88, 201801 (2002) arXiv:hep-ph/0201206; C. Anastasiou and K. Melnikov, Nucl. Phys. B 646, 220 (2002) arXiv:hep-ph/0207004 ; V. Ravindran, J. Smith and W. L. van Neerven, Nucl. Phys. B 665, 325 (2003) arXiv:hep-ph/0302135.

[23] A. D. Martin, R. G. Roberts, W. J. Stirling and R. S. Thorne, Eur. Phys. J. C 23, 73 (2002) arXiv:hep-ph/0110215; Phys. Lett. B 531, 216 (2002) arXiv:hep-ph/0201127; Eur. Phys. J. C 28, 455 (2003) arXiv:hep-ph/0211080.
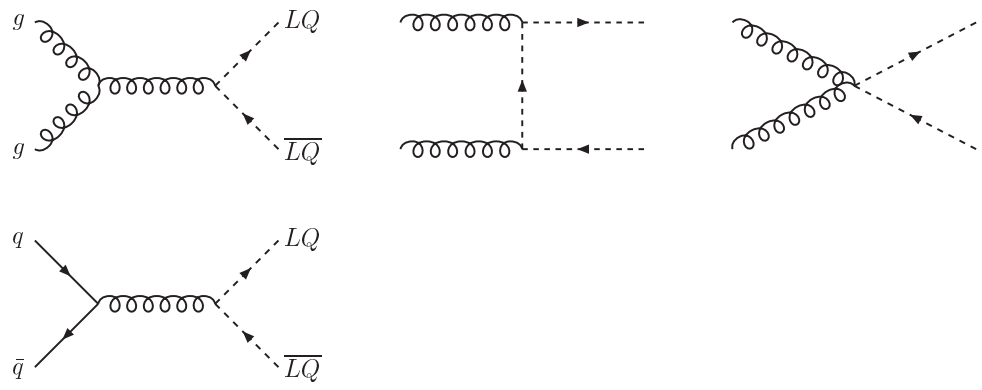

FIG. 1: Generic set of leading-order Feynman diagrams for leptoquark pair production through gluon-gluon fusion and quark-antiquark annihilation. 


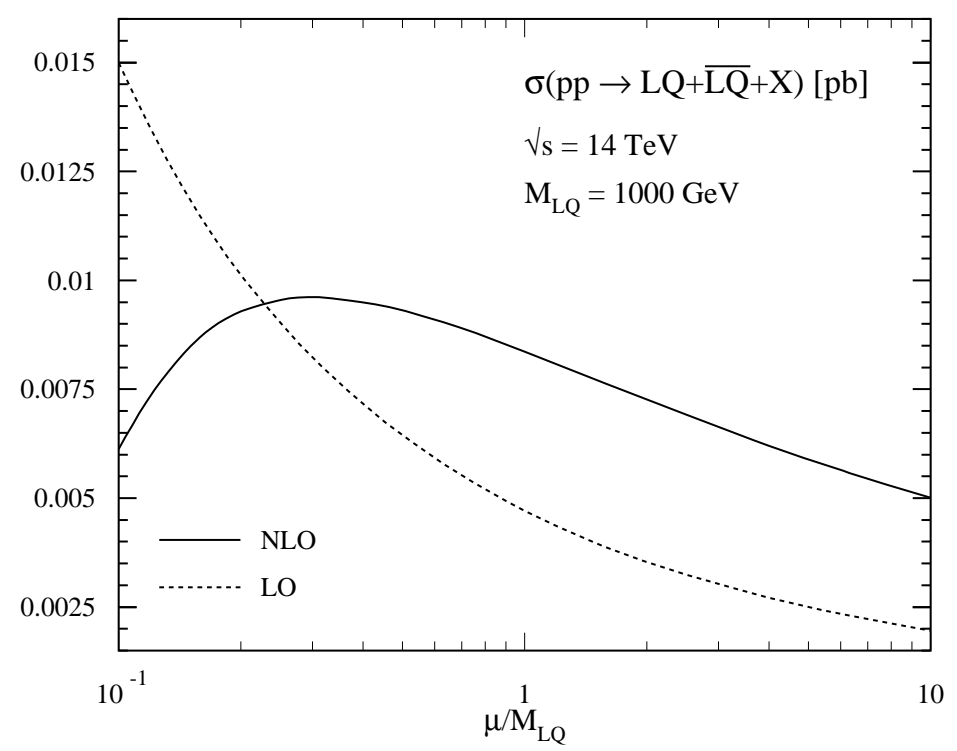

FIG. 2: Variation of the LO and NLO cross sections for $p p \rightarrow L Q+\overline{L Q}+X$ at the LHC with the renormalization and factorization scales. The leptoquark mass has been set to $M_{L Q}=1 \mathrm{TeV}$.

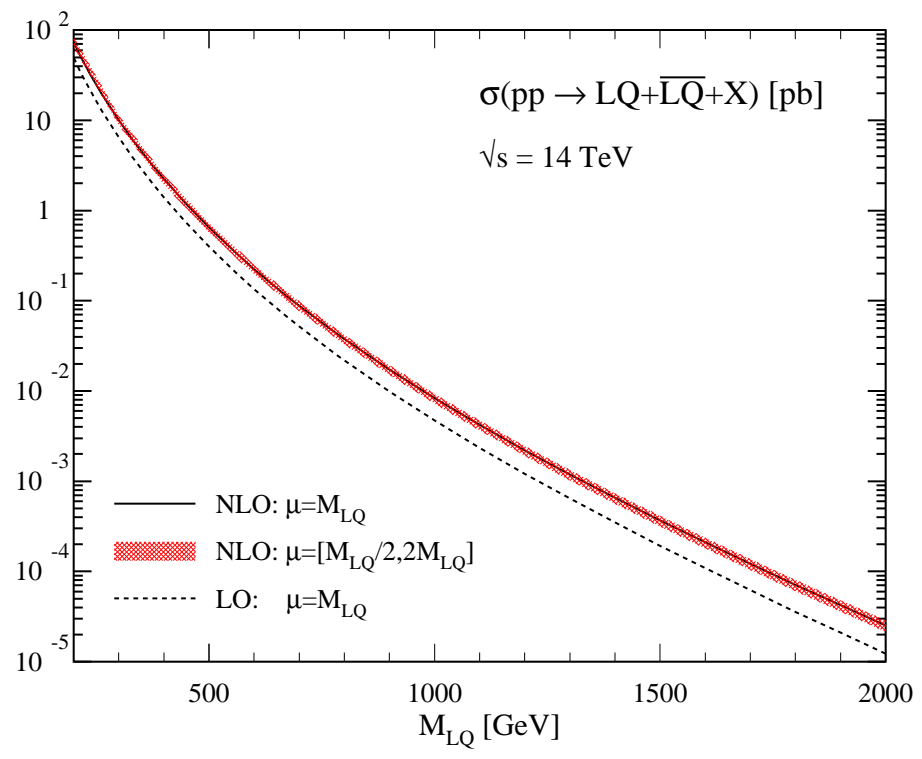

FIG. 3: Total cross section for $p p \rightarrow L Q+\overline{L Q}+X$ at the LHC energy $\sqrt{s}=14 \mathrm{TeV}$ as a function of the leptoquark mass $M_{L Q}$. The variation of the NLO cross section with the value of the renormalization/factorization scale is indicated by the shaded band. 
TABLE I: Total cross section and $K$-factors for $p p \rightarrow L Q+\overline{L Q}+X$ at the LHC energy $\sqrt{S}=14 \mathrm{TeV}$. The renormalization and factorization scales have been set to $\mu=M_{L Q}$. The CTEQ6L1 and CTEQ6M 14] parton densities have been adopted as default, whereas in the rightmost column a comparison is performed with the MRST2002 23] parton densities. The relative weight of $g g$, $q \bar{q}$ and $g q$ contributions to the cross section at NLO is given in the second-to-last column. [The negative sign of $\sigma_{g q}$ is a mere artifact of subtracting collinear initial-state singularities via mass factorization; by definition, this particular higher-order quantity must not be interpreted as a cross section.]

\begin{tabular}{|c||c|c|c|c||c|}
\hline \multicolumn{1}{|c||}{$M_{L Q}[\mathrm{GeV}]$} & \multicolumn{4}{|c||}{ CTEQ6 $(\mathrm{LO} / \mathrm{NLO})$} & MRST2002 \\
\cline { 2 - 6 } & $\sigma_{\mathrm{LO}}[\mathrm{fb}]$ & $\sigma_{\mathrm{NLO}}[\mathrm{fb}]$ & $g g: q \bar{q}: g q$ & $\mathrm{~K}$ & $\sigma_{\mathrm{NLO}}[\mathrm{fb}]$ \\
\hline \hline 200 & $0.500 \times 10^{2}$ & $0.742 \times 10^{2}$ & $0.94: 0.05: 0.01$ & 1.48 & $0.779 \times 10^{2}$ \\
400 & $0.140 \times 10^{1}$ & $0.224 \times 10^{1}$ & $0.91: 0.10:-0.01$ & 1.60 & $0.243 \times 10^{1}$ \\
600 & 0.135 & 0.225 & $0.88: 0.15:-0.03$ & 1.67 & 0.245 \\
800 & $0.219 \times 10^{-1}$ & $0.378 \times 10^{-1}$ & $0.84: 0.19:-0.03$ & 1.73 & $0.406 \times 10^{-1}$ \\
1000 & $0.471 \times 10^{-2}$ & $0.836 \times 10^{-2}$ & $0.82: 0.22:-0.04$ & 1.77 & $0.879 \times 10^{-2}$ \\
1200 & $0.121 \times 10^{-2}$ & $0.221 \times 10^{-2}$ & $0.81: 0.24:-0.05$ & 1.83 & $0.226 \times 10^{-2}$ \\
1400 & $0.349 \times 10^{-3}$ & $0.655 \times 10^{-3}$ & $0.79: 0.26:-0.05$ & 1.88 & $0.650 \times 10^{-3}$ \\
1600 & $0.109 \times 10^{-3}$ & $0.210 \times 10^{-3}$ & $0.78: 0.28:-0.06$ & 1.93 & $0.201 \times 10^{-3}$ \\
1800 & $0.357 \times 10^{-4}$ & $0.713 \times 10^{-4}$ & $0.77: 0.29:-0.06$ & 2.00 & $0.656 \times 10^{-4}$ \\
2000 & $0.122 \times 10^{-4}$ & $0.253 \times 10^{-4}$ & $0.77: 0.30:-0.07$ & 2.07 & $0.222 \times 10^{-4}$ \\
\hline
\end{tabular}

